\title{
Diffusion Coefficients of Electronically Excited Molecules
}

\author{
Alexander Sharipov, Boris Loukhovitski, Alexey Pelevkin \\ Central Institute of Aviation Motors, Moscow, 111116, Russia \\ assharipov@ciam.ru
}

\begin{abstract}
The work is devoted to the study of the influence of electronic excitation of molecules on their diffusion coefficients. Based on the electrical properties of several molecules $\left(\mathrm{O}_{2}, \mathrm{OH}, \mathrm{CO}, \mathrm{N}_{2}\right.$, $\mathrm{H}_{2} \mathrm{O}$, and $\mathrm{HO}_{2}$ ) in various electronic states known from literature and obtained by the authors using quantum chemical calculations, the binary diffusion coefficients on the main components of atmospheric air $\left(\mathrm{N}_{2}, \mathrm{O}_{2}, \mathrm{H}_{2} \mathrm{O}\right.$, and $\left.\mathrm{Ar}\right)$ were estimated over a wide temperature range. It is shown that the diffusion coefficients of electronically excited molecules can differ significantly from the diffusion coefficients of these molecules in the ground electronic state, especially for high excitation energies, as well as in the case of diffusion of a polar molecule in a polar buffer gas.
\end{abstract}

Keywords: electronically excited states, polarizability, dipole moment, collisional diameter, diffusion coefficient.

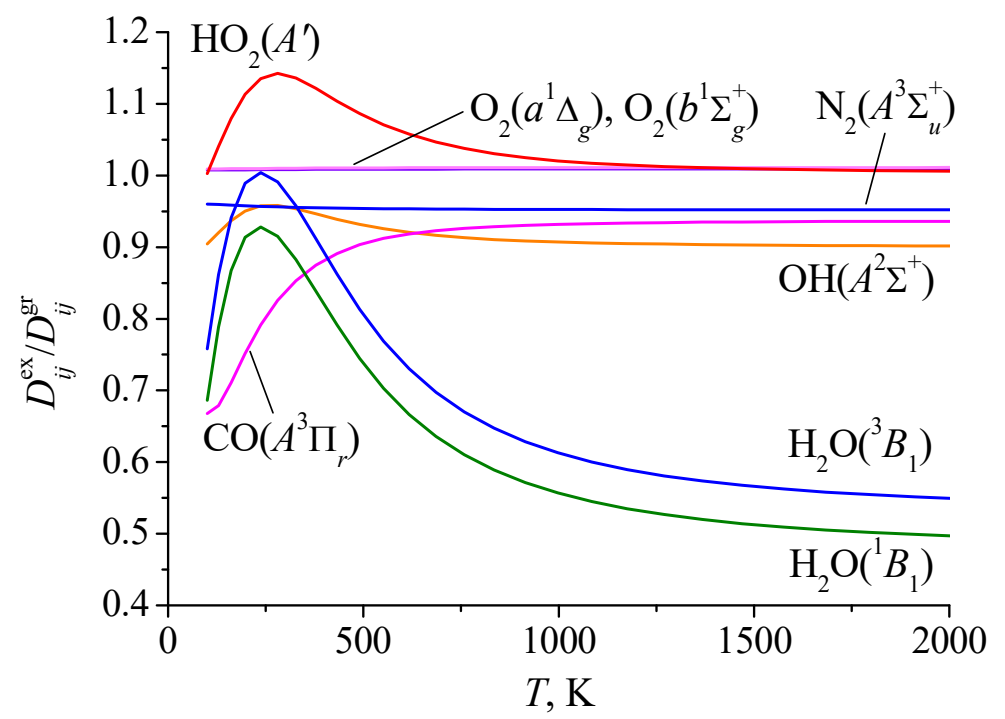

The ratio of the diffusion coefficients of electronically excited molecules to the diffusion coefficients of their counterparts in the ground electronic state (buffer gas is $\mathrm{H}_{2} \mathrm{O}$ ) 
УДК $533.1+539.194$

\title{
Коэффициенты диффузии электронно-возбужденных молекул
}

\author{
А. С. Шарипов, Б. И. Луховицкий, А. В. Пелевкин \\ Центральный институт авиационного моторостроения им. П.И. Баранова, \\ Россия, Москва, 111116, ул. Авиамоторная, д. 2 \\ assharipov@ciam.ru
}

\begin{abstract}
Аннотация
Работа посвящена исследованию влияния электронного возбуждения молекул на их коэффициенты диффузии. На основе известных и полученных авторами методами квантовой химии электрических свойств ряда молекул $\left(\mathrm{O}_{2}, \mathrm{OH}, \mathrm{CO}, \mathrm{N}_{2}, \mathrm{H}_{2} \mathrm{O}\right.$ и $\left.\mathrm{HO}_{2}\right)$ в различных электронных состояниях рассчитаны бинарные коэффициенты диффузии на основных компонентах атмосферного воздуха $\left(\mathrm{N}_{2}, \mathrm{O}_{2}, \mathrm{H}_{2} \mathrm{O}\right.$ и $\left.\mathrm{Ar}\right)$ в широком диапазоне температур. Показано, что коэффициенты диффузии электронно-возбужденных молекул могут существенно отличаться от коэффициентов диффузии этих молекул в основном электронном состоянии, особенно для больших энергий возбуждения, а также в случае диффузии полярной молекулы в полярном буферном газе.
\end{abstract}

Ключевые слова: электронно-возбужденные состояния, поляризуемость, дипольный момент, столкновительный диаметр, коэффициент диффузии.

\section{1. Введение}

Необходимость в определении свойств переноса возбужденных атомов и молекул возникает при решении различных задач физической газовой динамики, в частности, при моделировании термически неравновесных кинетических процессов за сильными ударными волнами (УВ) и в сверхзвуковых расширяющихся струях [1-5], в верхних слоях атмосферы различных планет [6], в равновесной и неравновесной плазме электрического разряда $[7,8]$, в задачах лазерно- [9] и плазменно-стимулированного горения $[10,11]$, а также при расчетах свойств высокотемпературного реагирующего газа [12-14].

Известно, что если молекулы находятся в возбужденных квантовых состояниях (вращательных, колебательных, электронных), то их электрические свойства, определяющие межмолекулярные потенциалы и сечения столкновения, отличаются от их аналогов в основных состояниях [15-17]. Поэтому возбуждение молекул в общем случае может привести к изменению их коэффициентов переноса по сравнению с невозбужденными компонентами $[16,18]$.

Нужно сказать, что влиянию данных эффектов при моделировании термически неравновесных физико-химических процессов до недавнего времени уделялось не слишком много внимания, хотя ряд исследований в прошлом был посвящен оценке свойств переноса колебательно- и вращательно- [18-21] возбужденных молекул и влиянию их надлежащего учета на процессы переноса вещества, импульса и энергии в различных газодинамических течениях [2-5]. В то же время зависимость коэффициентов переноса атомарных и молекулярных компонентов от степени их электронного возбуждения изучена гораздо слабее. По всей видимости это связано с тем, что такая зависимость обычно считается слабой для диффузии и вязкости и заметной лишь для теплопроводности [22, 23]. Так в ряде исследований, посвященных моделированию неравновесной плазмы разряда и её воздействию на процессы горения, за недостатком данных транспортные свойства электронно-возбужденных состояний 
считались такими же, как и у основных состояний соответствующих компонентов [24-27] или незначительно отличающимися с учетом изменений в межъядерном расстоянии [28].

Проблема отсутствия литературных данных по коэффициентам переноса электронновозбужденных компонентов усугубляется тем, что для их определения требуется знание соответствующих межмолекулярных потенциалов, непосредственное теоретическое определение которых весьма проблематично даже на современном уровне развития химической физики и квантовой химии. Лишь для коэффициентов переноса электронно-возбужденных атомарных компонентов развиты феноменологические подходы, основанные на полуклассическом квантово-механическом описании природы электронного возбуждения [13, 22, 29-32].

Что касается свойств переноса молекулярных компонентов, то здесь имеющиеся литературные данные гораздо более скудны, а последовательные теоретические подходы к их определению в условиях электронного возбуждения до сих пор не выработаны. Так, насколько известно авторам настоящей статьи, лишь в работах $[8,11,33]$ оценивалось влияние электронного возбуждения на коэффициент диффузии молекулярного кислорода. Однако систематического исследования влияния электронного возбуждения на процессы переноса молекул до сих пор проведено не было.

Данная работа ставит своей задачей восполнить этот пробел и представить методологию, позволяющую определять транспортные свойства электронно-возбужденных молекул на основе результатов неэмпирических квантово-химических расчетов. Использование предлагаемой методологии будет проиллюстрировано на примере оценки коэффициентов диффузии некоторых молекул, представляющих значение для химии горения и воздушной разрядной плазмы.

\section{2. Методология}

\section{1. Оцекнка коэффициентов диффузии}

Традиционная кинетическая теория газов позволяет оценить транспортные свойства отдельных молекул, если известен потенциал межчастичного взаимодействия. Бинарный коэффициент диффузии $D_{i j}$ может быть рассчитан с использованием стандартного выражения, полученного на основе теории Чепмена - Энскога [34]. Это уравнение, применимое при низких и умеренных давлениях, выглядит следующим образом [34]:

$$
D_{i j}=\frac{3}{8} \frac{\sqrt{2 \pi M_{i j} k T / N_{A}}}{\pi \sigma_{i j}^{2}} \frac{1}{\Omega^{(1,1)^{*}}} \frac{R T}{P_{0} 2 M_{i j}}
$$

Здесь $M_{i j}$ - приведенная молярная масса частиц сорта $i$ и $j ; \sigma_{i j}$ - столкновительный диаметр сталкивающихся частиц; $P_{0}$ - нормальное атмосферное давление; $R$ - универсальная газовая постоянная; $\Omega^{(1,1)^{*}}$ - приведенный интеграл столкновений, нормированный на сечение для жестких сфер диаметром $\sigma_{i j}$ и зависящий от температуры газа и межчастичного потенциала $\varphi_{i j}(r)$. В случае, если этот потенциал может быть сведен к виду сферически симметричного потенциала типа Леннарда-Джонса (ЛД), значения $\Omega^{(1,1)^{*}}\left(\varepsilon_{i j}, T\right)$, зависящие от эффективной потенциальной глубины ямы $\left(\varepsilon_{i j} / k_{b} T\right)$, могут быть рассчитаны, например, в соответствии с аппроксимационной формулой [35].

Известные в литературе данные о межмолекулярных потенциалах обычно имеют отношение к молекулам в основных квантовых состояниях. Однако если молекулы находятся в возбужденном состоянии, их столкновительные и электрические свойства отличаются от их аналогов в основном состоянии, и потенциал должен быть соответственно изменен. В общем случае данные о межмолекулярных потенциалах могут быть непосредственно получены из экспериментов по рассеянию молекулярных пучков [34], однако в связи с трудностями проведения соответствующих экспериментов с возбужденными молекулами (обусловленными 
их нестабильностью и невозможностью получения в макроскопических количествах) теоретическое определение межмолекулярных потенциалов возбужденных молекул имеет особе значение для моделирования газокинетических процессов.

Межмолекулярное взаимодействие неполярных нейтральных молекул зачастую описывается сферически симметричным потенциалом ЛД 12-6, описывающим прежде всего дисперсионное взаимодействие [36]. В то же время для полярных молекул также необходимо учитывать члены, зависящие от взаимной ориентации взаимодействующих частиц, ответственные за ориентационное (диполь-дипольное) и индукционное (диполь-наведенный диполь) взаимодействия [34].

В общем случае взаимодействие двух молекул $(i$ и $j)$ может быть описано в соответствии с методом приведенного диполя (МПД), разработанным в работе [37]. В рамках МПД потенциал Штокмайера 12-6-3 с ориентационными членами, часто используемый для аппроксимации взаимодействия произвольных молекул, посредством термически-равновесного усреднения по ориентациям может быть сведен к эффективному сферически симметричному потенциалу ЛД 12-6 [38]. В соответствии с данным подходом взаимодействие между парой полярных (в общем случае) молекул на расстоянии $r$ определяется потенциалом

$$
\phi_{i j}^{\text {eff }}(r)=C_{6}^{\text {eff }}\left(\frac{\left(\sigma_{i j}^{\text {eff }}\right)^{6}}{r^{12}}-\frac{1}{r^{6}}\right)
$$

со следующими эффективными параметрами:

$$
\begin{aligned}
& C_{6}^{\mathrm{eff}}=C_{6}^{\mathrm{disp}}\left(1+\frac{C_{6}^{\mathrm{ind}}}{C_{6}^{\mathrm{disp}}}+\frac{C_{6}^{\mathrm{el}}}{4 C_{6}^{\mathrm{disp}}}\right)^{2}, \\
& \sigma_{i j}^{\mathrm{eff}}=\sigma_{i j}\left(1+\frac{C_{6}^{\mathrm{ind}}}{C_{6}^{\mathrm{disp}}}+\frac{C_{6}^{\mathrm{el}}}{4 C_{6}^{\text {disp }}}\right)^{-1 / 6},
\end{aligned}
$$

где $\sigma_{i j}$ - столкновительный диаметр частиц, а $C_{6}{ }^{\mathrm{disp}}, C_{6}{ }^{\mathrm{el}}$ и $C_{6}{ }^{\text {ind }}-$ члены, определяющие дисперсионное, электростатическое (диполь-дипольное) взаимодействие диполей и индукционное (диполь-наведенный диполь) взаимодействие, соответственно. Зависимость этих членов от дипольных моментов $\mu$ и поляризуемости $\alpha$ сталкивающихся молекул записывается стандартным образом согласно [38]. Однако для этого требуется определить соответствующие значения для молекул в электронно-возбужденных состояниях.

\section{2. Электрические свойства электронно-возбужденных молекул}

Электрические свойства молекул в возбужденных электронных состояниях гораздо менее известны и реже изучаются (как экспериментально, так и теоретически), чем их аналоги в основном состоянии $[17,39]$, несмотря на то, что эти свойства в последние годы вызывают все больший интерес в различных областях $[11,23,33,39]$. Дело в том, что поляризуемость молекул в возбужденных электронных состояниях может быть совершенно иной, чем в основных состояниях [40], в результате чего такие молекулы будут обладать, в частности, особыми транспортными свойствами. Знание поляризуемости возбужденных состояний также имеет значение для оптической (главным образом, лазерной) диагностики возбужденных частиц в неравновесных газовых потоках [17]. Наконец, основные молекулярные электрические свойства, такие как дипольные моменты и дипольные поляризуемости в возбужденных электронных состояниях дают очень важную информацию об особенностях электронной структуры этих состояний [39] и, соответственно, об их реакционной способности.

Отметим, что теоретическая оценка электрических свойств молекул в возбужденных электронных состояниях является достаточно проблематичной задачей даже для современной квантовой химии. Дело в том, что лишь немногие из расчетных методов квантовой химии дают 
возможность рассматривать электронное возбуждение. Возбужденные электронные состояния требуют ухода от однодетерминантного описания и часто демонстрируют значимость статических и динамических корреляционных эффектов [39, 40]. В результате, для точного определения электрических свойств таких молекул должны быть использованы современные квантово-химические методы, которые учитывают многодетерминантный характер волновой функции и эффекты электронной корреляции, такие как многоконфигурационный метод самосогласованного поля и метод связанных кластеров.

В Таблице 1 приведены значения статической электронной поляризуемости и дипольного момента для основных и некоторых возбужденных электронных состояний для ряда молекул, таких как $\mathrm{O}_{2}, \mathrm{OH}, \mathrm{CO}, \mathrm{N}_{2}, \mathrm{H}_{2} \mathrm{O}$ и $\mathrm{HO}_{2}$, представляющих значение как для собственно кинетики плазменно-стимулированного горения [41, 42], так и для задач оптической диагностики неравновесных газовых потоков $[43,44]$. Представленные значения получены в рамках высокоточных квантово-химических расчетах в работах $[17,40,45]$, а также в ходе расчетов в рамках данной работы (для $\mathrm{OH}, \mathrm{CO}$ и $\mathrm{N}_{2}$ ). В последнем случае расчеты проводились с помощью метода конечного поля на уровне теории XMCQDPT2/d-aug-cc-pVQZ (см. описание методологии в [45]) с помощью программы FireFly [46]. Для молекул $\mathrm{OH}, \mathrm{CO}$ и $\mathrm{N}_{2}$ использовались активные пространства следующих размеров: $(7,5),(10,8)$ и $(10,8)$, здесь первая цифра показывает число электронов в активном пространстве, а вторая - число орбиталей, на которых они распределены.

Заметим, что использование данной методологии для описания электрических свойств молекул было обосновано путем сравнения с экспериментальными данными для кислорода, полученными методом дифракции на лазерно-индуцированных решетках в [17]. Кроме того, полученное в наших расчетах значение дипольного момента $\operatorname{CO}\left(a^{3} \Pi_{r}\right)$ весьма близко к экспериментальному значению 1.374 D [47], полученному методом штарковской спектроскопии.

\section{Таблица 1}

Электрические свойства и газокинетический диаметр рассматриваемых компонентов

\begin{tabular}{|c|c|c|c|c|c|c|}
\hline Молекула & $T_{\mathrm{e}}$, эВ & $\alpha, \AA^{3}$ & $\mu, \mathrm{D}$ & Источник & $\sigma_{i i}, \AA$ & Источник \\
\hline $\mathrm{O}_{2}\left(X^{3} \Sigma_{g}^{-}\right)$ & 0 & 1.584 & - & {$[17]$} & 3.407 & {$[37]$} \\
\hline $\mathrm{O}_{2}\left(a^{1} \Delta_{g}\right)$ & 0.98 & 1.534 & - & {$[17]$} & 3.371 & оценка \\
\hline $\mathrm{O}_{2}\left(b^{1} \Sigma_{g}^{+}\right)$ & 1.64 & 1.523 & - & {$[17]$} & 3.363 & оценка \\
\hline $\mathrm{OH}\left(X^{2} \Pi_{i}\right)$ & 0 & 1.177 & 1.650 & расчет & 3.111 & {$[37]$} \\
\hline $\mathrm{OH}\left(A^{2} \Sigma^{+}\right)$ & 4.05 & 1.708 & 1.782 & расчет & 3.522 & оценка \\
\hline $\mathrm{CO}\left(X^{1} \Sigma^{+}\right)$ & 0 & 1.948 & 0.100 & расчет & 3.652 & {$[37]$} \\
\hline $\mathrm{CO}\left(a^{3} \Pi_{r}\right)$ & 6.04 & 2.451 & 1.398 & расчет & 3.943 & оценка \\
\hline $\mathrm{N}_{2}\left(X^{1} \Sigma_{g}^{+}\right)$ & 0 & 1.825 & - & расчет & 3.652 & {$[37]$} \\
\hline $\mathrm{N}_{2}\left(A^{3} \Sigma_{u}^{+}\right)$ & 6.22 & 2.181 & - & расчет & 3.856 & оценка \\
\hline $\mathrm{H}_{2} \mathrm{O}\left(X^{1} A_{1}\right)$ & 0 & 1.423 & 1.873 & {$[40]$} & 2.673 & {$[37]$} \\
\hline $\mathrm{H}_{2} \mathrm{O}\left({ }^{3} B_{1}\right)$ & 6.83 & 11.228 & 0.628 & {$[40]$} & 5.321 & оценка \\
\hline $\mathrm{H}_{2} \mathrm{O}\left({ }^{1} B_{1}\right)$ & 7.26 & 15.574 & 1.090 & {$[40]$} & 5.935 & оценка \\
\hline $\mathrm{HO}_{2}\left(A^{\prime \prime}\right)$ & 0 & 2.027 & 2.241 & {$[45]$} & 3.433 & {$[37]$} \\
\hline $\mathrm{HO}_{2}\left(A^{\prime}\right)$ & 0.87 & 1.997 & 1.773 & {$[45]$} & 3.416 & оценка \\
\hline $\mathrm{Ar}$ & 0 & 1.642 & - & {$[36]$} & 3.350 & {$[36]$} \\
\hline
\end{tabular}


Как можно видеть, электронное возбуждение в целом приводит к существенному увеличению поляризуемости молекул, хотя в некоторых случаях $\left(\mathrm{O}_{2}\right.$ и $\left.\mathrm{HO}_{2}\right)$ поляризуемость возбужденных состояний даже слегка меньше значений поляризуемости основного состояния. Что касается влияния на дипольный момент, знак эффекта электронного возбуждения также может быть различным.

\section{3. Столкновительный диаметр электронно-возбужденных молекул}

Считается, что линейный размер электронной плотности атома или молекулы примерно пропорционален $\alpha^{1 / 3}$, где $\alpha$ - статическая поляризуемость компонента $[48,49]$. Этот факт целесообразно использовать для модификации газокинетических диаметров, достаточно надежно известных для молекул в основных электронных состояниях, с целью учесть влияние электронного возбуждения. Заметим, что в основе известного в литературе подхода к оценке транспортных свойств возбужденных систем через так называемый радиус Слэтера [29] и применимого лишь для высоковозбужденных атомарных компонентов [32], лежит та же самая связь поляризуемости с линейным масштабом системы.

В Таблице 1 приведены значения газокинетического диаметра для молекул $\mathrm{O}_{2}, \mathrm{OH}, \mathrm{CO}$, $\mathrm{N}_{2}, \mathrm{H}_{2} \mathrm{O}$ и $\mathrm{HO}_{2}$ в основном электронном состоянии по данным работы [37], а также оценки значений $\sigma_{i i}$ в случае электронного возбуждения этих молекул, сделанные путем пересчета пропорционально кубическому корню соответствующих поляризуемостей. Видим, что согласно используемой методологии электронное возбуждение в целом может приводить к заметному увеличению столкновительного диаметра молекул, в особенности при больших энергиях электронного возбуждения $T_{\mathrm{e}}$.

Для оценки столкновительных диаметров, необходимых для определения коэффициентов бинарной диффузии, следуя [38], использовалось эмпирическое комбинационное правило Бастьена [50].

\section{3. Результаты и обсуждение}

На основе данных, приведенных в Таблице 1 для рассматриваемых молекул в различных состояниях были рассчитаны коэффициенты бинарной диффузии на каждом из основных компонентов атмосферного воздуха $\left(\mathrm{N}_{2}, \mathrm{O}_{2}, \mathrm{H}_{2} \mathrm{O}, \mathrm{Ar}\right)$ в широком диапазоне температур при атмосферном давлении.

Стоит отметить, что выражение для коэффициентов бинарной диффузии (1) хоть и, строго говоря, справедливо лишь для разреженных газов, в действительности его точность относительно более строгих приближений кинетической теории газов вполне достаточна для большинства практических приложений. Так, оценки влияния числовой плотности молекул на коэффициенты диффузии по модели твердых сфер [51] при $T=1000 \mathrm{~K}$ дают погрешность относительно диффузии в разреженном газе около $0.1 \%$ для $P=1$ атм и $1.2 \%$ для $P=100$ атм. Погрешность используемых аппроксимаций интегралов столкновений [35] также весьма невелика (в пределах $0.1 \%$ ). Подробнее о расчете коэффициентов переноса с учетом высших приближений метода Чепмена - Энскога см., например, в [52].

Что касается неточности, связанной с использованием МПД-формализма (выражения (2)-(4)) относительно более точных моделей межмолекулярных взаимодействий, то какиелибо непредвзятые числовые оценки получить весьма затруднительно в силу того, что более сложные модели требуют задания дополнительных и в общем случае неизвестных входных параметров. Однако из сравнения с экспериментальными данными для коэффициентов переноса можно заключить, что при температурах свыше $300 \div 400 \mathrm{~K}$ МПД дает точность по крайне мере не худшую, чем другие популярные подходы [36, 38].

Наконец, существенным источником неопределенности при расчете коэффициентов диффузии электронно-возбужденных молекул согласно методологии настоящей работы яв- 
ляется погрешность при определении электрических ( $\mu$ и $\alpha)$ и газокинетических $\left(\sigma_{i i}\right)$ параметров молекул. Так из сравнения рассчитанных в настоящей работе значений дипольного момента основного и возбужденного состояний с экспериментальными данными [47] типичную величину $\Delta \mu$ примем равной $0.02 \mathrm{D}$. Из аналогичного сравнения для молекулярного кислорода [17]типичную величину $\Delta \alpha$ для основного и возбужденного состояний возьмем равной $0.02 \AA^{3}$.Что касается погрешности определения столкновительных диаметров молекул в основном электронном состоянии, заимствованных из работы [37], то согласно критическому анализу, проведенному в [36], $\Delta \sigma_{i i}$ в данном случае можно оценить как $0.2 \AA$.

Оценим погрешность вычисления коэффициента диффузии $\Delta D_{i j}$ как сложной функции от переменных $\alpha, \mu$ и $\sigma_{i i}$, считая остальные величины идеально определенными, см. выражение (1). Для вышеприведенных значений погрешности определения значений поляризуемости, дипольного момента и столкновительного диаметра $\left(\Delta \alpha=0.02 \AA^{3}, \Delta \mu=0.02 D\right.$, $\left.\Delta \sigma_{i i}=0.2 \AA\right)$, искомая погрешность $\Delta D_{i j}$ не превышает $5 \%$ для всего набора столкновительных партнеров в диапазоне температур от 273 до $6000 \mathrm{~K}$. Однако, как будет видно далее, для многих комбинаций столкновительных партнеров изменение коэффициента диффузии в результате электронного возбуждения молекулы может быть порядка или даже меньше 5 \% и, таким образом, вычисление абсолютных значений коэффициентов диффузии для таких электронно-возбужденных молекул с использованием предложенной методологии теряет смысл. Однако известно, что современные экспериментальные и теоретические подходы позволяют оценить изменение электрических свойств молекул в результате электронного возбуждения с большей точностью, чем для самого значения электрического свойства [53], т.е., например, если для молекулы $\mathrm{O}_{2}$ поляризуемость известна с точностью $0.02 \AA^{3}(1.2 \%)$, то точность определения отношения поляризуемости $\mathrm{O}_{2}\left(b^{1} \Sigma_{g}^{+}\right)$к поляризуемости $\mathrm{O}_{2}\left(X^{3} \Sigma_{g}^{-}\right)$составляет уже $0.6 \%[17]$.

В связи с этим имеет смысл представить полученные результаты в виде отношения коэффициента бинарной диффузии $i$-й молекулы в электронно-возбужденном состоянии в $j$-м буферном газе $D_{i j}^{\mathrm{ex}}$ к аналогичному коэффициенту для основного состояния $D_{i j}^{\mathrm{gr}}$. Это отношение будем аппроксимировать в диапазоне температур $T=273 \div 6000 \mathrm{~K}$ согласно [52] в виде

$$
\frac{D_{i j}^{\mathrm{ex}}(T)}{D_{i j}^{\mathrm{gr}}(T)}=\exp \left[p_{1}+p_{2} \ln (T)+p_{3}(\ln (T))^{2}+p_{4}(\ln (T))^{3}\right],
$$

где $i$ - компонент, для которого рассматривается электронное возбуждение, а $j$ - газ-разбавитель $\left(\mathrm{N}_{2}, \mathrm{O}_{2}, \mathrm{H}_{2} \mathrm{O}\right.$ или $\left.\mathrm{Ar}\right)$. Полученные в ходе аппроксимации коэффициенты приведены в Таблице 2.

Таблица 2

Коэффициенты аппроксимации температурных зависимостей $D_{i j}{ }^{\mathrm{ex}} / D_{i j}{ }^{\mathrm{gr}}$ в различных буферных газах

\begin{tabular}{|l|l|l|l|l|}
\hline \multicolumn{1}{|c|}{$i$ на $j$} & $p_{1}$ & \multicolumn{1}{c|}{$p_{2}$} & $p_{3}$ & $p_{4}$ \\
\hline $\mathrm{O}_{2}\left(a^{1} \Delta_{g}\right)$ на $\mathrm{N}_{2}\left(X^{1} \Sigma_{g}^{+}\right)$ & $1.48 \mathrm{E}-03$ & $2.66 \mathrm{E}-03$ & $-3.34 \mathrm{E}-04$ & $1.40 \mathrm{E}-05$ \\
\hline $\mathrm{O}_{2}\left(a^{1} \Delta_{g}\right)$ на $\mathrm{O}_{2}\left(X^{3} \Sigma_{g}^{-}\right)$ & $9.61 \mathrm{E}-02$ & $-3.42 \mathrm{E}-02$ & $4.25 \mathrm{E}-03$ & $-1.76 \mathrm{E}-04$ \\
\hline $\mathrm{O}_{2}\left(a^{1} \Delta_{g}\right)$ на $\mathrm{H}_{2} \mathrm{O}\left(X^{1} A_{1}\right)$ & $-1.88 \mathrm{E}-02$ & $9.96 \mathrm{E}-03$ & $-1.19 \mathrm{E}-03$ & $4.70 \mathrm{E}-05$ \\
\hline $\mathrm{O}_{2}\left(a^{1} \Delta_{g}\right)$ на $\mathrm{Ar}$ & $-2.29 \mathrm{E}-03$ & $4.14 \mathrm{E}-03$ & $-5.17 \mathrm{E}-04$ & $2.15 \mathrm{E}-05$ \\
\hline $\mathrm{O}_{2}\left(b^{1} \Sigma_{g}^{+}\right)$на $\mathrm{N}_{2}\left(X^{1} \Sigma_{g}^{+}\right)$ & $1.96 \mathrm{E}-03$ & $3.20 \mathrm{E}-03$ & $-4.02 \mathrm{E}-04$ & $1.68 \mathrm{E}-05$ \\
\hline $\mathrm{O}_{2}\left(b^{1} \Sigma_{g}^{+}\right)$на $\mathrm{O}_{2}\left(X^{3} \Sigma_{g}^{-}\right)$ & $9.49 \mathrm{E}-02$ & $-3.31 \mathrm{E}-02$ & $4.11 \mathrm{E}-03$ & $-1.70 \mathrm{E}-04$ \\
\hline
\end{tabular}


Продолжение Таблиць 2

\begin{tabular}{|c|c|c|c|c|}
\hline$i$ на $j$ & $p_{1}$ & $p_{2}$ & $p_{3}$ & $p_{4}$ \\
\hline $\mathrm{O}_{2}\left(b^{1} \Sigma_{g}^{+}\right)$на $\mathrm{H}_{2} \mathrm{O}\left(X^{1} A_{1}\right)$ & $-2.27 \mathrm{E}-02$ & $1.21 \mathrm{E}-02$ & $-1.44 \mathrm{E}-03$ & $5.71 \mathrm{E}-05$ \\
\hline $\mathrm{O}_{2}\left(b^{1} \Sigma_{g}^{+}\right)$на $\mathrm{Ar}$ & $-2.64 \mathrm{E}-03$ & $5.00 \mathrm{E}-03$ & $-6.24 \mathrm{E}-04$ & $2.59 \mathrm{E}-05$ \\
\hline $\mathrm{OH}\left(A^{2} \Sigma^{+}\right)$на $\mathrm{N}_{2}\left(X^{1} \Sigma_{g}^{+}\right)$ & $8.46 \mathrm{E}-02$ & $-6.87 \mathrm{E}-02$ & 8.63E-03 & $-3.61 E-04$ \\
\hline $\mathrm{OH}\left(A^{2} \Sigma^{+}\right)$на $\mathrm{O}_{2}\left(X^{3} \Sigma_{g}^{-}\right)$ & $1.68 \mathrm{E}-01$ & $-9.95 \mathrm{E}-02$ & $1.24 \mathrm{E}-02$ & $-5.14 \mathrm{E}-04$ \\
\hline $\mathrm{OH}\left(A^{2} \Sigma^{+}\right)$на $\mathrm{H}_{2} \mathrm{O}\left(X^{1} A_{1}\right)$ & $2.01 \mathrm{E}+00$ & $-7.66 \mathrm{E}-01$ & $9.25 \mathrm{E}-02$ & $-3.72 \mathrm{E}-03$ \\
\hline $\mathrm{OH}\left(A^{2} \Sigma^{+}\right)$на $\mathrm{Ar}$ & $1.41 \mathrm{E}-01$ & $-9.02 \mathrm{E}-02$ & $1.13 \mathrm{E}-02$ & $-4.67 \mathrm{E}-04$ \\
\hline $\mathrm{CO}\left(a^{3} \Pi_{r}\right)$ на $\mathrm{N}_{2}\left(X^{1} \Sigma_{g}^{+}\right)$ & $-1.57 \mathrm{E}-01$ & $3.60 \mathrm{E}-02$ & $-4.54 \mathrm{E}-03$ & $1.90 \mathrm{E}-04$ \\
\hline $\mathrm{CO}\left(a^{3} \Pi_{r}\right)$ на $\mathrm{O}_{2}\left(X^{3} \Sigma_{g}^{-}\right)$ & $-1.02 \mathrm{E}-01$ & $1.51 \mathrm{E}-02$ & $-1.89 \mathrm{E}-03$ & $7.86 \mathrm{E}-05$ \\
\hline $\mathrm{CO}\left(a^{3} \Pi_{r}\right)$ на $\mathrm{H}_{2} \mathrm{O}\left(X^{1} A_{1}\right)$ & $-6.44 \mathrm{E}+00$ & $2.48 \mathrm{E}+00$ & $-3.20 \mathrm{E}-01$ & $1.37 \mathrm{E}-02$ \\
\hline $\mathrm{CO}\left(a^{3} \Pi_{r}\right)$ на $\mathrm{Ar}$ & $-1.47 \mathrm{E}-01$ & $3.14 \mathrm{E}-02$ & $-3.94 \mathrm{E}-03$ & $1.64 \mathrm{E}-04$ \\
\hline $\mathrm{N}_{2}\left(A^{3} \Sigma_{u}^{+}\right)$на $\mathrm{N}_{2}\left(X^{1} \Sigma_{g}^{+}\right)$ & $-1.32 \mathrm{E}-01$ & $3.50 \mathrm{E}-02$ & $-4.44 \mathrm{E}-03$ & $1.87 \mathrm{E}-04$ \\
\hline $\mathrm{N}_{2}\left(A^{3} \Sigma_{u}^{+}\right)$на $\mathrm{O}_{2}\left(X^{3} \Sigma_{g}^{-}\right)$ & 2.97E-02 & $-2.87 \mathrm{E}-02$ & $3.61 \mathrm{E}-03$ & $-1.51 \mathrm{E}-04$ \\
\hline $\mathrm{N}_{2}\left(A^{3} \Sigma_{u}^{+}\right)$на $\mathrm{H}_{2} \mathrm{O}\left(X^{1} A_{1}\right)$ & 8.86E-02 & $-5.00 \mathrm{E}-02$ & $6.04 \mathrm{E}-03$ & $-2.43 \mathrm{E}-04$ \\
\hline $\mathrm{N}_{2}\left(A^{3} \Sigma_{u}^{+}\right)$на $\mathrm{Ar}$ & $1.15 \mathrm{E}-02$ & $-2.22 \mathrm{E}-02$ & $2.80 \mathrm{E}-03$ & $-1.17 \mathrm{E}-04$ \\
\hline $\mathrm{H}_{2} \mathrm{O}\left({ }^{3} B_{1}\right)$ на $\mathrm{N}_{2}\left(X^{1} \Sigma_{g}^{+}\right)$ & $2.32 \mathrm{E}+00$ & $-1.06 \mathrm{E}+00$ & $1.31 \mathrm{E}-01$ & $-5.36 \mathrm{E}-03$ \\
\hline $\mathrm{H}_{2} \mathrm{O}\left({ }^{3} B_{1}\right)$ на $\mathrm{O}_{2}\left(X^{3} \Sigma_{g}^{-}\right)$ & $2.93 \mathrm{E}+00$ & $-1.28 \mathrm{E}+00$ & $1.56 \mathrm{E}-01$ & $-6.37 \mathrm{E}-03$ \\
\hline $\mathrm{H}_{2} \mathrm{O}\left({ }^{3} B_{1}\right)$ на $\mathrm{H}_{2} \mathrm{O}\left(\mathrm{X}^{1} \mathrm{~A}_{1}\right)$ & $5.74 \mathrm{E}+00$ & $-1.48 \mathrm{E}+00$ & 7.63E-02 & $1.14 \mathrm{E}-03$ \\
\hline $\mathrm{H}_{2} \mathrm{O}\left({ }^{3} B_{1}\right)$ на $\mathrm{Ar}$ & $2.81 \mathrm{E}+00$ & $-1.24 \mathrm{E}+00$ & $1.51 \mathrm{E}-01$ & $-6.16 \mathrm{E}-03$ \\
\hline $\mathrm{H}_{2} \mathrm{O}\left({ }^{1} B_{1}\right)$ на $\mathrm{N}_{2}\left(X^{1} \Sigma_{g}^{+}\right)$ & $2.79 \mathrm{E}+00$ & $-1.27 \mathrm{E}+00$ & $1.58 \mathrm{E}-01$ & $-6.49 \mathrm{E}-03$ \\
\hline $\mathrm{H}_{2} \mathrm{O}\left({ }^{1} B_{1}\right)$ на $\mathrm{O}_{2}\left(X^{3} \Sigma_{g}^{-}\right)$ & $3.47 \mathrm{E}+00$ & $-1.52 \mathrm{E}+00$ & $1.87 \mathrm{E}-01$ & $-7.63 \mathrm{E}-03$ \\
\hline $\mathrm{H}_{2} \mathrm{O}\left({ }^{1} B_{1}\right)$ на $\mathrm{H}_{2} \mathrm{O}\left(X^{1} A_{1}\right)$ & $5.86 \mathrm{E}+00$ & $-1.53 \mathrm{E}+00$ & 7.92E-02 & $1.17 \mathrm{E}-03$ \\
\hline $\mathrm{H}_{2} \mathrm{O}\left({ }^{1} B_{1}\right)$ на $\mathrm{Ar}$ & $3.33 \mathrm{E}+00$ & $-1.47 \mathrm{E}+00$ & $1.81 \mathrm{E}-01$ & $-7.39 \mathrm{E}-03$ \\
\hline $\mathrm{HO}_{2}\left(A^{\prime}\right)$ на $\mathrm{N}_{2}\left(X^{1} \Sigma_{g}^{+}\right)$ & $2.98 \mathrm{E}-01$ & $-1.09 \mathrm{E}-01$ & $1.34 \mathrm{E}-02$ & $-5.47 \mathrm{E}-04$ \\
\hline $\mathrm{HO}_{2}\left(A^{\prime}\right)$ на $\mathrm{O}_{2}\left(X^{3} \Sigma_{g}^{-}\right)$ & $2.96 \mathrm{E}-01$ & $-1.07 \mathrm{E}-01$ & $1.30 \mathrm{E}-02$ & $-5.29 \mathrm{E}-04$ \\
\hline $\mathrm{HO}_{2}\left(A^{\prime}\right)$ на $\mathrm{H}_{2} \mathrm{O}\left(X^{1} A_{1}\right)$ & $3.74 \mathrm{E}+00$ & $-1.31 \mathrm{E}+00$ & $1.51 \mathrm{E}-01$ & $-5.81 \mathrm{E}-03$ \\
\hline $\mathrm{HO}_{2}\left(A^{\prime}\right)$ на $\mathrm{Ar}$ & $3.27 \mathrm{E}-01$ & $-1.18 \mathrm{E}-01$ & $1.45 \mathrm{E}-02$ & $-5.88 \mathrm{E}-04$ \\
\hline
\end{tabular}

Чтобы проанализировать полученные результаты, рассмотрим рассчитанные температурные зависимости, взяв в качестве примера столкновительных партнеров неполярную молекулу $\mathrm{N}_{2}$ (см. рис. $1, a$ ) и молекулу $\mathrm{H}_{2} \mathrm{O}$, обладающую существенным дипольным моментом (см. рис. $1, b$ ). Видим, что в случае возбуждения молекул в сравнительно невысоко лежащие электронные состояния, такие как $\mathrm{O}_{2}\left(a^{1} \Delta_{g}\right), \mathrm{O}_{2}\left(b^{1} \Sigma_{g}^{+}\right)$и $\mathrm{HO}_{2}\left(A^{\prime}\right)\left(T_{\mathrm{e}}<2\right.$ эВ), коэффициенты диффузии в неполярных газах почти не меняются (слегка возрастают) по сравнению с молекулами в основном электронном состоянии. Однако коэффициенты диффузии молекул в более высоколежащих электронных состояниях уже оказываются существенно меньшими, чем для невозбужденных молекул, что связанно с заметным ростом поляризуемости (и, как 
следствие, газокинетического сечения) при электронном возбуждении. При этом для отношений $D_{i j}^{\mathrm{ex}} / D_{i j}^{\mathrm{gr}}$ наблюдается довольно слабая температурная зависимость.

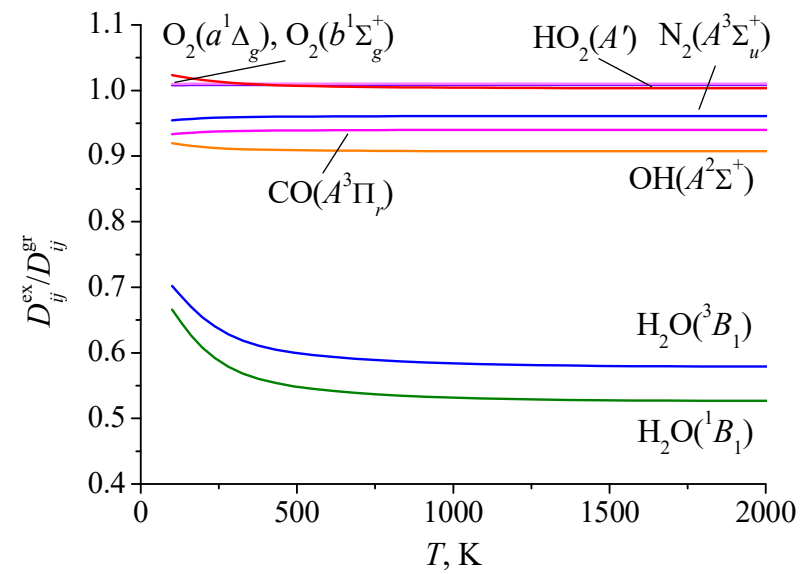

$a$

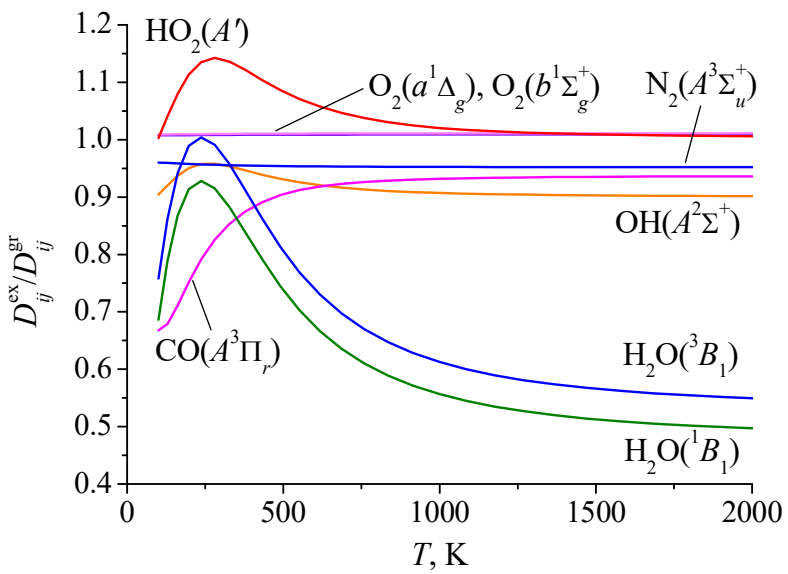

$b$

Рис. 1. Температурная зависимость отношения коэффициента диффузии различных электронновозбужденных молекул на $\mathrm{N}_{2}(a)$ и $\mathrm{H}_{2} \mathrm{O}(b)$ к аналогичному коэффициенту для тех же молекул в основном состоянии при $P=1$ атм

Что касается диффузии в полярном газе, как видно из рис. $1 b$ на примере водяного пара в качестве разбавителя, при низких температурах $(T<1000 \mathrm{~K})$ существенное влияние на диффузию оказывает зависимость дипольного момента молекулярного компонента от степени электронного возбуждения $T_{\mathrm{e}}$. Это приводит к тому,что для электронно-возбужденных молекул с заметным дипольным моментом отношение $D_{i j}{ }^{\text {ex }} / D_{i j}{ }^{\text {gr }}$ может иметь сильную и даже немонотонную температурную зависимость.

\section{4. Выводы}

С учетом знания электрических свойств ряда молекул $\left(\mathrm{O}_{2}, \mathrm{OH}, \mathrm{CO}, \mathrm{N}_{2}, \mathrm{H}_{2} \mathrm{O}\right.$ и $\left.\mathrm{HO}_{2}\right)$ в различных электронных состояниях, полученных в ходе многоконфигурационных квантовохимических расчетов данной работы и других авторов, для широкого диапазона температур были рассчитаны бинарные коэффициенты диффузии на основных компонентах атмосферного воздуха $\left(\mathrm{N}_{2}, \mathrm{O}_{2}, \mathrm{H}_{2} \mathrm{O}\right.$ и $\left.\mathrm{Ar}\right)$. При этом параметры межмолекулярных потенциалов рассчитывались в соответствии с методом приведенного диполя с учетом дисперсионного, диполь-дипольного и индукционного взаимодействий.

Было выявлено, что электронное возбуждение молекул может приводить к существенному влиянию на коэффициенты диффузии, в особенности для больших энергий возбуждения, а также в случае диффузии полярной молекулы в полярном буферном газе.

Представленная методология дает возможность проводить соответствующие оценки для любых молекул, для электронно-возбужденных состояний которых имеются или могут быть получены надежные данные по основным электрическим свойствам (поляризуемость, дипольный момент).

\section{Благодарности}

Работа выполнена при финансовой поддержке РФФИ (проект № 20-38-70014). 


\section{References}

1. Adamovich, I. V., Macheret, S. O., Rich, J. W., "Spatial nonhomogeneity effects in nonequilibrium vibrational kinetics," Chem. Phys., Vol. 182, 1994, pp. 167-183.

2. Galkin, V. S., Makashev, N. K., Rastigeev, E. A., "Estimation of the effect of vibrational excitation of diatomic molecules on their diffusional transport and dissociation in a boundary layer," Fluid Dyn., Vol. 31, 1996, pp. 144-155.

3. Kustova, E. V., Nagnibeda, E. A., "Transport properties of a reacting gas mixture with strong vibrational and chemical nonequilibrium," Chem. Phys., Vol. 233, 1998, pp. 57-75.

4. Armenise, I., Barbato, M., Capitelli, M., Kustova, E., "State-to-state catalytic models, kinetics, and transport in hypersonic boundary layers," J. Thermophys. Heat Transfer, Vol. 20, 2006, pp. 465-476.

5. Kremer, G. M., Kunova, O. V., Kustova, E. V., Oblapenko, G. P., "The influence of vibrational stateresolved transport coefficients on the wave propagation in diatomic gases," Physica A., Vol. 490, 2018, pp. 92-113.

6. Campbell, L., Brunger, M. J., "Modelling of plasma processes in cometary and planetary atmospheres," Plasma Sources Sci. Technol., Vol. 22, 2013, p. 013002 (34 pp).

7. Capitelli, M., Armenise, I., Bisceglie, E., Bruno, D., Celiberto, R., Colonna, G., D'Ammando, G., Pascale, O. D., Esposito, F., Gorse, C., Laporta, V., Laricchiuta, A., "Thermodynamics, transport and kinetics of equilibrium and non-equilibrium plasmas: A state-to-state approach," Plasma Chem. Plasma Process, Vol. 32, 2012, pp. 427-450.

8. Azyazov, V. N., Torbin, A. P., Pershin, A. A., Mikheyev, P. A., Heaven, M. C., "Kinetics of oxygen species in an electrically driven singlet oxygen generator," Chem. Phys., Vol. 463, 2015, pp. 65-69.

9. Tropina, A. A., New-Tolley, M. R., Shneider, M. N., "Modeling of laser ignition in hydrogen-air mixture," AIAA SciTech 2020 Forum, 2020, p. 1892.

10. Tropina, A. A., Uddi, M., Ju, Y., "On the effect of nonequilibrium plasma on the minimum ignition energy: Part 2," IEEE T. Plasma Sci., Vol. 39, 2011, pp. 3283-3287.

11. Pineda, D. I., Chen, J. Y., "Effects of updated transport properties of singlet oxygen species on steady laminar flame simulations," Western States Section Spring Technical Meeting of the Combustion Institute, Seattle, WA, 2016, Paper 139LF-0021.

12. D'Angola, A., Colonna, G., Gorse, C., Capitelli, M., "Thermodynamic and transport properties in equilibrium air plasmas in a wide pressure and temperature range," Eur. Phys. J. D., Vol. 46, 2008, pp. 129-150.

13. Capitelli, M., Bruno, D., Colonna, G., Catalfamo, C., Laricchiuta, A., "Thermodynamics and transport properties of thermal plasmas: the role of electronic excitation," J. Phys. D: Appl. Phys., Vol. 42, 2009, p. 194005.

14. Wang, W., Wu, Y., Rong, M., Ehn, L., Cernusak, I., "Theoretical computation of thermophysical properties of high-temperature $\mathrm{F}_{2}, \mathrm{CF}_{4}, \mathrm{C}_{2} \mathrm{~F}_{2}, \mathrm{C}_{2} \mathrm{~F}_{4}, \mathrm{C}_{2} \mathrm{~F}_{6}, \mathrm{C}_{3} \mathrm{~F}_{6}$ and $\mathrm{C}_{3} \mathrm{~F}_{8}$ plasmas," J. Phys. D: Appl. Phys., Vol. 45, 2012, p. 285201.

15. Loukhovitski, B. I., Sharipov, A. S., Starik, A. M., "Influence of vibrations and rotations of diatomic molecules on their physical properties: I. Dipole moment and static dipole polarizability," J. Phys. B: At. Mol. Opt. Phys., Vol. 49, 2016, p. 125102.

16. Sharipov, A. S., Loukhovitski, B. I., Starik A. M., "Influence of vibrations of polyatomic molecules on dipole moment and static dipole polarizability: theoretical study," J. Phys. B: At. Mol. Opt. Phys., Vol. 50, 2017, p. 165101 (19 pp).

17. Sharipov, A. S., Loukhovitski, B. I., Pelevkin, A. V., Kobtsev, V. D., Kozlov D.N., "Polarizability of electronically excited molecular oxygen: theory and experiment," J. Phys. B: At. Mol. Opt. Phys., Vol. 52, 2019, p. 045101. 
18. Sharipov, A. S., Loukhovitski, B. I., Starik, A. M., "Influence of vibrations and rotations of diatomic molecules on their physical properties: II. Refractive index, diffusion coefficients, reactivity," J. Phys. B: At. Mol. Opt. Phys., Vol. 49, 2016, p. 125103.

19. Kunc, J. A., "Central-force potentials for interaction of rotationally and vibrationally excited molecules," J. Phys. B: At. Mol. Opt. Phys., Vol. 23, 1990, pp. 2553-2566.

20. Kang, S. H., Kunc, J. A., "Molecular diameters in high-temperature gases," J. Phys. Chem. Vol. 95, 1991, pp. 6971-6973.

21. Gorbachev, Y. E., Gordillo-Vaszquez, F. J., Kunc, J. A., "Diameters of rotationally and vibrationally excited diatomic molecules," Physica A, Vol. 247, 1997, pp. 108-120.

22. Capitelli, M., Celiberto, R., Gorse, C., Laricchiuta, A., Minelli, P., Pagano, D., "Electronically excited states and transport properties of thermal plasmas: The reactive thermal conductivity," Phys. Rev. E., Vol. 66, 2002, p. 016403.

23. Kustova, E. V., Puzyreva, L. A., "Transport coefficients in nonequilibrium gas-mixture flows with electronic excitation," Phys. Rev. E, Vol. 80, 2009, p. 046407.

24. Gordiets, B., Ferreira, C. M., Pinheiro, M. J., Ricard, A., "Self-consistent kinetic model of low-pressure $\mathrm{N}_{2}-\mathrm{H}_{2}$ flowing discharges: I. Volume processes," Plasma Sources Sci. Technol., Vol. 7, 1998, pp. 363-378.

25. Bourig, A., Thevenin, D., Martin, J.-P., Janiga, G., Zahringer, K., "Numerical modeling of $\mathrm{H}_{2}-\mathrm{O}_{2}$ flames involving electronically-excited species $\mathrm{O}_{2}\left(a^{1} \Delta_{g}\right), \mathrm{O}\left({ }^{1} \mathrm{D}\right)$ and $\mathrm{OH}\left({ }^{2} \Sigma^{+}\right)$, , Proc. Combust. Inst., Vol. 32, 2009, pp. 3171-3179.

26. Konnov, A. A., "On the role of excited species in hydrogen combustion," Combust. Flame, Vol. 162, 2015, pp. 3755-3772.

27. Ombrello, T., Popov, N., "Mechanisms of ethylene flame propagation enhancement by $\mathrm{O}_{2}\left(a^{1} \Delta_{g}\right)$," Aerospace Lab., 2015, hal-01270947

28. Kozlov, V. E., Starik, A. M., Titova, N. S., "Enhancement of combustion of a hydrogen-air mixture by excitation of $\mathrm{O}_{2}$ molecules to the $a^{1} \Delta_{g}$ state," Combust. Expl. Shock Waves, Vol. 44, 2008, pp. 371379.

29. Hirschfelder, J. O., Eliason, M. A., "The estimation of the transport properties for electronically excited atoms and molecules," Annals New York Acad. Sci., Vol. 67, No. 9, 1957, pp. 451-461

30. Eletskii, A. V., Capitelli, M., Celiberto, R., Laricchiuta, A., "Resonant charge exchange and relevant transport cross sections for excited states of oxygen and nitrogen atoms," Phys. Rev. A, Vol. 69, 2004, p. 042718.

31. Istomin, V. A., Kustova, E. V., Mekhonoshina, M. A., "Eucken correction in high-temperature gases with electronic excitation," J. Chem. Phys., Vol. 140, 2014, p. 184311.

32. Istomin, V. A., Kustova, E. V., "State-specific transport properties of partially ionized flows of electronically excited atomic gases," Chem. Phys., Vol. 485, 2017, pp. 125-139.

33. Kobtsev, V. D., Kostritsa, S. A., Smirnov, V. V., Titova, N. S., Torokhov, S. A., "Flow reactor experimental study of $\mathrm{H}_{2} / \mathrm{O}_{2}$ and $\mathrm{H}_{2} /$ air mixtures ignition assisted by the electrical discharge," Combust. Sci. Technol., Vol. 192, 2020, pp. 744-759.

34. Hirschfelder, J. O., Curtiss, C. F., Bird, R. B., Molecular theory of gases and liquids, John Wiley and sons, NY; Chapman and Hall, London, 1954.

35. Neufeld, P. D., Janzen, A.R., Aziz, R. A., "Empirical equations to calculate 16 of the transport collision integrals $\Omega^{(l, s)^{*}}$ for the Lennard-Jones (12-6) potential," J. Chem. Phys., Vol. 57, 1972. pp. 1100-1103.

36. Brown, N. J., Bastien, L. A., Price, P. N., "Transport properties for combustion modeling," Prog. Energy Combust. Sci., Vol. 37, 2011, pp. 565-582.

37. Paul, P., Warnatz, J., "A re-evaluation of the means used to calculate transport properties of reacting flows,” Proc. Combust. Inst., Vol. 27, 1998, pp. 495-504. 
38. Sharipov, A. S., Loukhovitski, B. I., Tsai, C.-J., Starik, A. M., "Theoretical evaluation of diffusion coefficients of $\left(\mathrm{Al}_{2} \mathrm{O}_{3}\right)_{n}$ clusters in different bath gases," Eur. Phys. J. D., Vol. 68, 2014, p. 99.

39. Ruud, K., Mennucci, B., Cammi, R., Frediani, L., "The calculation of excited-state polarizabilities of solvated molecules," J. Comput. Methods Sci. Eng., Vol. 4, 2004, pp. 381-397.

40. Paleníková, J., Kraus, M., Neogrády, P., Kellö, V., Urban, M., "Theoretical study of molecular properties of low-lying electronic excited states of $\mathrm{H}_{2} \mathrm{O}$ and $\mathrm{H}_{2} \mathrm{~S}$," Mol. Phys., Vol. 106, 2008. pp. 2333 2344.

41. Starik, A. M., Loukhovitski, B. I., Sharipov, A. S., Titova, N. S., "Physics and chemistry of the influence of excited molecules on combustion enhancement," Phil. Trans. R. Soc. A., Vol. 373, 2015, p. 20140341.

42. Starikovskiy, A., Aleksandrov, N., "Plasma-assisted ignition and combustion," Prog. Energy Combust. Sci., Vol. 39, 2013, pp. 61-110.

43. Eremin, A. V., Korshunova, M. R., Mikheyeva, E. Y., "Influence of flame suppressants on the level of nonequilibrium radiation during ignition of hydrogen-oxygen mixtures behind shock waves," Combust. Expl. Shock Waves, Vol. 55, 2019, pp. 121-124.

44. Bystrov, N., Emelianov, A., Eremin, A., Loukhovitski, B., Sharipov, A., Yatsenko, P., "Experimental study of high temperature oxidation of dimethyl ether, n-butanol and methane," Combust. Flame, Vol. 218, 2020, pp. 121-133.

45. Pelevkin, A. V., Sharipov, A. S., "Interaction of $\mathrm{CH}_{4}$ with electronically excited $\mathrm{O}_{2}$ : ab initio potential energy surfaces and reaction kinetics," Plasma Chem. Plasma Process, Vol. 39, 2019, pp. 1533-1558.

46. Granovsky, A. A. Firefly, V., 8.2.0 http://classic.chem.msu.su/gran/firefly/index.html

47. Wicke, B. G., Klemperer, W., "Experimental dipole moment function and calculated radiative lifetimes for vibrational transitions in carbon monoxide $a^{3} \Pi^{*}, " J$. Chem. Phys., Vol. 63, 1975, pp. 37563763.

48. Cambi, R., Cappelletti, D., Liuti, G., Pirani, F., "Generalized correlations in terms of polarizability for van der Waals interaction potential parameter calculations," J. Chem. Phys., Vol. 95, 1991, pp. 1852 1862.

49. Hohm, U., Thakkar, A. J., "New relationships connecting the dipole polarizability, radius, and second ionization potential for atoms," J. Phys. Chem. A., Vol. 116, 2012, pp. 697-703.

50. Bastien, L. A. J., Price, P. N., Brown, N. J., "Intermolecular potential parameters and combining rules determined from viscosity data," Int. J. Chem. Kinet., Vol. 42, 2010, pp. 713-723.

51. Dymond, J. H., "Hard-sphere theories of transport properties," Chem. Soc. Rev., Vol. 14, 1985, pp. 317-356.

52. Sokolova, I. A., Vasiljevskii, S. A., Andriatis, A. V., "Calculation of transport coefficients of multicomponent gas and plasma," Physical-Chemical Kinetics in Gas Dynamics, Vol. 3, 2005. http://chemphys.edu.ru/issues/2005-3/articles/80/

53. Pelevkin, A. V., Kadochnikov, I. N., Sharipov, A. S., "Theoretical study of the interaction of atomic nitrogen with electronically excited molecular oxygen," Physical-Chemical Kinetics in Gas Dynamics, Vol. 20, No. 2, 2019. http://chemphys.edu.ru/issues/2019-20-2/articles/837/

54. Middha, P., Yang, B., Wang, H., "A first-principle calculation of the binary diffusion coefficients pertinent to kinetic modeling of hydrogen/oxygen/helium flames," Proc. Combust. Inst. Vol. 29, 2002, pp. 1361-1369.

Статья поступила в редакцию 26 ноября 2020 г. 